\title{
Journal of Architectural Research and Development
}

Review Article

\section{Inheritance and Evolution of the Spatial Form of Traditional Rural Settlement}

\author{
Lianying $\mathrm{Li}^{1,2}$, Dawei $\mathrm{Xu}^{1 *}$, Xi Chen ${ }^{1}$, Safa Fadelelseed ${ }^{1}$
}

${ }^{1}$ School of Landscape Architecture, Northeast Forestry University, Harbin, 150040, P. R. China.

${ }^{2}$ Harbin University, Harbin, 150086, P. R. China.

\begin{abstract}
As early as the farming era, traditional rural settlements have been deeply rooted in our country. The geographical location of rural settlements is inextricably linked to the natural environment, transportation and social forms. China has continuously increased its modernization and transformed rural settlements with the rapid development of society, which has led to the change in the spatial form of rural settlements and led to the demise of many traditional settlements. Therefore, the culture and traditions are inevitably deplorable. Settlement is the most primitive way of living in China. Therefore, it is necessary to understand and learn the culture as well as correctly transform the rural settlement. This paper aims to study the spatial form of traditional rural settlements. Based on the background research of traditional rural settlement spatial form, this paper discusses the influencing factors of settlement spatial form and the spatial form and evolution of rural settlements.
\end{abstract}

Keywords: Traditional Village, Rural Settlement, Spatial Form

Publication date: September, 2019

Publication online: 30 September 2019

*Corresponding author: Dawei Xu, xudw@nefu.edu.cn

\section{Background research of the spatial form of traditional rural settlement}

Influenced by the new ideas of Western human geography in the 1930s, the study of human geography in China began to take off. The development of rural settlements had opportunities for development ${ }^{[1]}$. Plus, the rural settlement geography as a branch of human geography has also developed rapidly with the influx of more and more researchers. "Traditional villages", "aggregate places", "rural settlements" and "villagers gathering points" have become hot words in humanities research. Many researchers have become interested in rural settlements in China and have made unremitting efforts to settle our rural areas. The map and historical trajectory are improved step by step, which is convenient for contemporary scholars to understand the development pulse and development status of China's rural settlement. In addition, from the research, we can divide the development of rural settlements in China into three major stages.

The first stage: Reform and opening to the early 1990s. During this period of time, China has implemented a policy of opening to the outside world and people's ideas have been further liberated. At the same time, domestic culture can conduct diversified exchanges with the outside world. The academic trend is relatively high, and the thinking is more active. A large number of scholars have begun to investigate rural settlements.

The second stage: From the early 1990s to the end of the 1990s. At the end of the 90s, the main residents of China are still dominated by rural residents and the study of rural settlements has become a very important research task. The economic income of farmers has undergone earth-shaking changes with the in-depth development of reform and opening up, which has attracted more researchers' attention. The primary consideration of researchers on "Why rural settlements have developed so rapidly?" and "How to upgrade them?" have become the study of rural settlements and it has entered a stage of rapid development.

The third stage: From the late 1990s to the present 
is the diversified research stage of traditional rural settlements. After 2000, the problems in rural areas were widely valued by the government. The government proposed to accelerate the modernization of rural areas and promote the rapid development of new rural areas. Therefore, more scholars have participated in the study of traditional rural settlements. Thus, the content of the study was further expanded and entered a period of diversification.

\section{Constituent elements of the settlement space form}

In order to study the spatial form of rural settlements, we must first understand the spatial elements that make up the settlement.

\subsection{Formal external elements: boundaries}

The marginal matter of the rural settlement forms the boundary of the settlement. Understanding from the perspective of the constituent elements, the boundaries of settlements can be divided into artificial boundaries, natural boundaries and mixed boundaries ${ }^{[2]}$. Artificial boundaries refer to artificially designed buildings that were used to divide the boundaries such as boundary pillars. Natural boundaries refer to naturally formed boundaries such as cliffs, lakes, lakes, and etc. while mixed boundaries are formed by artificial factors and natural factors. Through studying the boundaries of rural settlements in China, it was easily concluded that that the boundaries of rural settlements in China are mostly made up of mixed boundaries.

In addition, all three borders are physical boundaries. Therefore, for residents in rural settlements, the boundaries tend to be more of a spatial concept. The settlement is a virtual and solid relationship as a whole and the internal physical buildings, physical passages as well as external boundaries constitute a settlement together. The interior of the settlement consists of a number of small plaques that extend continuously from the road to the boundary of the settlement. Besides, from this point of view, the foundation of this virtual and real relationship is the line and the surface, the line is the road and the surface are the building group. This relationship exists widely in the interior of the settlement to the edge of the settlement.

\subsection{Internal elements of form: roads and buildings}

The internal elements of the settlement mainly include roads and buildings. The road is the bones of the entire settlement, while the buildings are the meat of the settlement. The roads and buildings together constitute the internal environment of the entire village settlement. The building group generally uses the road as a reference for planning and construction. In other words, the construction of the road is also affected by the building community.

The road carries the circulation of information and materials within a settlement and the transportation of materials and information between the outside and the outside. Therefore, road construction is very important for rural settlements.

In the rural settlements, the roads are mainly dry. Besides, the buildings are mostly laid on both sides of the road and distributed radially in the direction of the road. The more prosperous the road, the more compact the building complex; on the contrary, the more rugged the road and the looser the building clusters nearby. This kind of circle-type feature is the embodiment of the hierarchical characteristics of a self-organizing system. This feature should be analyzed from the spatial occupancy of the building group, the stability of the building group and the connectivity of the building group in the specific quantitative expression $^{[3]}$.

\subsection{The influence of nonlinear relationship between boundary, road and building group on the shape of settlement space}

The borders, roads and buildings form a complete village together. The boundary defines the spatial extent of the rural settlements while the roads and buildings form the internal space of the rural settlement. In addition, it is said that the building complex is the basis of the boundary. The boundary of the settlement is actually intangible. As time goes by, the development of the complex and the influx and migration of the population as well as the settlement can become larger. Thus, the boundary can be expanded outward and it may become smaller and shrink the boundary inward.

The building group does not only affect the construction of the road but also determines the boundary of the settlement. The size of the boundary also determines the scale of development of the settlement while vice versa that the size of the settlement affects the scale of the complex.

The size of the settlement boundary depends on the number of buildings within the settlement and the proportion of the space of the settlement such as the number of buildings, the density and the connectivity between the buildings together determine the boundary 
of the settlement ${ }^{[4]}$.

Besides, when the building complex develops to a certain stage, the existing road facilities cannot meet the flow of population and material. Therefore, it is necessary to rebuild the road. The construction of the road will support the development of the economy and eventually the economic development will promote the population growth. In addition, from this point of view, this is a benign cycle. Moreover, it can be said that the road is the main facility for settlements presents a development status of a settlement, which can positively promote the development of settlements and the construction of buildings.

Roads, buildings and settlement boundaries complement each other and influence each other. This is a factor that makes the spatial form of rural settlements change in the short term and stable for a long time ${ }^{[5]}$. The level of the road and the size of the settlement boundary are mutually reinforcing. In other words, the more concentrated the road is in the building group, the more compact the distribution of the building group, the smaller the settlement boundary range and the settlement space shape will tend to be a group.

\section{Spatial form of traditional rural settlement}

\subsection{Evolution of traditional rural settlement spatial form}

Researchers suggest that rural settlements have experienced three stages such as the traditional homogenization pattern, the heterogeneous hybrid form in the early stage of transformation and the functional block layout pattern in the late stage of transformation ${ }^{[6]}$.

In the morphological evolution stage, there are three types of settlement spatial form evolution, which can be separated as spread-like block expansion, band expansion and dispersion expansion along the original plaque.

The evolution of the rural settlement space is mainly reflected on the increasing of land area and density as well as more regular distribution of the shape of each plaque. Moreover, under the influence of high-intensity human development activities, the number of plaques and their density become larger, the average area of plaques becomes smaller and the standard deviation of maximum plaque index and plaque area decreases. Therefore, it is indicating that the spatial pattern of rural settlements is fragmented ${ }^{[7]}$.
In addition, the spatial distribution of settlements is also affected by the natural environment and economic conditions. In areas with harsher natural environments, the distribution of settlements is rarer. Conversely, there are more distributions of settlements in suitable environments. The natural environment is linked to the economic level to a certain extent, and the economic conditions are good. Therefore, it can attract more residents to gather together. The population growth will increase the power of transforming the environment and promote the development of the settlement.

\subsection{Factors affecting the evolution of spatial forms of traditional rural settlements}

There are three main factors that lead to the evolution of the spatial form of surviving settlements such as geographical environmental factors, socio-economic factors and policy systems.

Among many geographical factors, topography is the main factor that is affecting the spatial distribution of settlements. The number of settlements in the plains of China is significantly higher than that the basins and plateau hills. The smoother the geological environment, the better the distribution of settlements. In addition to the terrain, water is also an important factor affecting the distribution of settlements. As an example for farmers, water is not only a necessity for life, but also an indispensable resource for labor production. In order to facilitate production and production, many settlement residents will choose to establish settlements near the water source.

Among many factors of social and economic development, the economic level of the settlement and the transportation facilities have a great influence on the spatial form of the settlement. The higher the economic level, the larger the settlement and the more convenient the transportation will be. Secondly, the change of livelihood methods caused by the change of disposable resources of villagers is a deep-seated reason to promote the spatial pattern change of rural settlements ${ }^{[8]}$. The land was expropriated, and the village name was forced to change the mode of production, which led to changes in the living space and activities of the residents. This change also promoted the changes in the industrial structure and social form of the area, which then further led to changes in the spatial form of the settlement.

According to the research results from relevant scholars, the economic status of farmers, the level of education, the intensity of government subsidies and the degree of perfection of social security have a significant 
positive impact on the willingness to live. However, for the age, satisfaction with the infrastructure and purchasing of houses as well as its price are negatively related to the farmers' willingness to live together in new community ${ }^{[9]}$. Therefore, the more developed the economic settlement, the faster the transformation and spatial reconstruction of rural settlements. On the other hand, the settlement is difficult to implement and the reconstruction of settlement space is difficult to advance in areas where the economy is underdeveloped.

Besides, the policy system also affects the changes in the form of settlement space. As the country continues to strengthen its emphasis on rural modernization, the government has continuously issued relevant policies and reform documents and the development of settlements has changed from self-evolution to government-led development. The policies issued by the government and related systems have promoted the reconstruction of the settlement space. However, some scholars believe that this destroys the characteristics and traditions of the settlement space. Therefore, relevant scholars have proposed a synergistic symbiosis mechanism between planning space and autonomous space to ensure that the government planning space can be implemented while guiding disorderly spontaneous construction behavior ${ }^{[10]}$.

\section{Conclusion}

In summary, the constituent elements of the traditional rural settlement spatial form are borders, roads and buildings. The spatial form of rural settlements has undergone three stages of evolution. Besides, the evolution factors affecting the spatial form of rural settlements are mainly categorized into three aspects such as geographical environment factors, social and economic development factors and policy systems. Among the geographical factors, topographical factors and water source factors have the greatest impact on settlement space. Moreover, socio-economic factors are the main source of power to promote the reconstruction of rural settlements while policy system provides the development direction for the reconstruction of settlements. In conclusion, it is worth to mention all stated factors in promoting the development of the settlement and we must also respect the characteristics and traditions of the settlement.

Funded project: Funded by the project of the Key Projects of Art Science Planning in Heilongjiang Province entitled "Study on Visual Landscape Control of Outer Environment of Manchu Residences in North China." (No. 2018A015).

\section{References}

[1] Pu XC, Dong YF. A Summary of the Quantitative Research on the Traditional Rural Settlement Patterns in China[J]. Architecture and Culture, 2018(8):59-61.

[2] Dong YF. Quantitative Study on the Boundary Shape of Traditional Rural Settlements[D]. Zhejiang University, 2018.

[3] Li XH. Study on the rural settlement patterns in the Shashang District of Zhangjiagang City[D]. Suzhou University of Science and Technology, 2017.

[4] Dong S. Research on the support foundation of traditional village space construction in North China[D]. Beijing Jianzhu University, 2017.

[5] Wen Y. A Preliminary Study on the Inheritance and Evolution of the Spatial Form of Rural Settlements[D]. Tianjin University, 2017.

[6] Li S. Huizhou Rural Geography Research[D]. Anhui University, 2017.

[7] Xiao LY, Zhou GH, Tang CL, He YH, Gao LJ. A Review of the Study on Rural Settlement in China since Reform and Opening up[J].Western Journal of Habitat Environment, 2016, 31(06):79-85.

[8] Chen XH, Zhao YY. Recent Progress in the Study of Spatial Reconstruction of Rural Settlements in China[J]. Journal of Chizhou University, 2016, 30(06):44-9.

[9] Wen XT. Research on Self-organizing Spatial Morphology of Rural Settlements in Harbin[D]. Harbin Institute of Technology, 2016.

[10] He L. Modern Reconstruction of Rural Independent Construction Model[D]. Tianjin University, 2017. 